\title{
Serum levels of GDF15 are reduced in preeclampsia and the reduction is more
}

profound in late-onset than early-onset cases

Qi Chen ${ }^{1,2 *}$, Yao Wang ${ }^{3,4}$, Min Zhao ${ }^{5}$, Jonathan Hyett ${ }^{6,7}$, Fabricio da Silva Costa ${ }^{8}$ and Guiying Nie ${ }^{3,4,9}$

${ }^{1}$ The Hospital of Obstetrics \& Gynaecology, Fudan University, China;

${ }^{2}$ Department of Obstetrics \& Gynaecology, University of Auckland, New Zealand;

${ }^{3}$ Centre for Reproductive Health, Hudson Institute of Medical Research, Clayton, VIC, Australia;

${ }^{4}$ Department of Molecular and Translational Sciences, Monash University, Clayton, VIC, Australia;

${ }^{5}$ Wuxi Maternity and Children's Health Hospital, Nanjing Medical University, Jiangsu, China;

${ }^{6}$ Central Clinical School, University of Sydney, Sydney, NSW, Australia;

${ }^{7}$ RPA Women and Babies, Royal Prince Alfred Hospital, Sydney, NSW, Australia;

${ }^{8}$ Monash Ultrasound for Women, Melbourne, VIC, Australia

${ }^{9}$ Department of Biochemistry and Molecular Biology, Monash University, Australia

Running title: Serum GDF15 is reduced in preeclampsia

*Corresponding author: Qi Chen,

The Hospital of Obstetrics \& Gynaecology, Fudan University, China;

419 Fangxie Road, Shanghai, China

Ph: 8613611691734

Email: q.chen@auckland.ac.nz 


\begin{abstract}
Background: Preeclampsia is a pregnancy specific disorder affecting 3-5\% of pregnancies worldwide. It is clinically divided into early-onset and late-onset subtypes. Placental factors are involved in the pathogenesis of preeclampsia. Growth differentiation factor 15 (GDF15), a protein of the transforming growth factor beta superfamily, is highly expressed in the placenta. However, it is unclear whether the circulating levels of GDF15 are altered in preeclampsia at the time of or prior to disease presentation.
\end{abstract}

Methods: Serum samples across three trimesters from 29 healthy pregnancies, third trimester sera from 34 women presenting with preeclampsia (early-onset $n=16$, late-onset $n=18$ ) and 66 gestation-age-matched controls, and sera at 11-13 weeks of pregnancy from women who later did $(n=36)$ or did not $(n=33)$ develop late-onset preeclampsia, were examined for GDF15 by ELISA.

Results: Serum GDF15 levels increased significantly with gestation in normal pregnancy. Serum GDF15 was significantly reduced in the third trimester in women presenting with preeclampsia compared to their gestation-age-matched controls. This reduction was apparent in both early-onset and late-onset subtypes, but it was more profound in late-onset cases. At 11-13 weeks of gestation, however, serum levels of GDF15 were similar between women who subsequently did and did not develop late-onset preeclampsia.

Conclusion: Serum GDF15 increased with gestation age, reaching the highest level in the third trimester. Serum GDF15 was significantly reduced in the third trimester in women presenting with preeclampsia, especially in late-onset cases. However, serum GDF15 was not altered in the first trimester in women destined to develop late-onset preeclampsia.

Key Word: preeclampsia, serum, GDF15, early-onset and late-onset preeclampsia 


\section{Introduction}

Preeclampsia is a pregnancy-specific disorder that presents as new-onset maternal hypertension and proteinuria after 20 weeks of gestation. It affects 3-5\% of pregnancies worldwide and is a major cause of maternal and perinatal mortality and morbidity [1]. Characteristics of preeclampsia include maternal endothelial cell activation and exaggerated inflammation [2, 3]. Preeclampsia is clinically divided into early-onset $(<34$ weeks of gestation) and late-onset (>34 weeks of gestation) subtypes according to the gestation age of disease onset $[4,5]$; it's becoming increasingly clear that the two subtypes have distinct characteristics $[6,7]$. Although the causes of preeclampsia are not yet completely understood, it is well accepted that factors released from the placenta are involved in the pathogenesis of preeclampsia, and that hypoxia and oxidative stress increase placental production of these factors [8]. In addition, preeclampsia is associated with the risk of developing cardiovascular disease later in life [9], suggesting that preeclampsia and cardiovascular disease may share common risk factors such as those involved in endothelial dysfunction and exaggerated inflammatory responses $[10,11]$.

Growth differentiation factor 15 (GDF15), also known as macrophage inhibitory cytokine-1 (MIC-1), is a protein of the transforming growth factor beta (TGF $\beta$ ) superfamily that regulates inflammatory and apoptotic pathways in injured tissues and during disease processes [12]. GDF15 is a stress-induced cytokine and associated with hypoxia, inflammation and oxidative stress (review in [13]), it is also released from endothelial cells after stimulation with pro-inflammatory cytokines [14]. GDF15 has been suggested as a target and biomarker for cardiovascular disease as it plays a cardioprotective role in the adult heart $[13,15]$.

GDF15 is highly expressed in the placenta, and comparatively its expression is lower in other tissues such as the kidneys, pancreas, prostate and colon under physiological conditions [16- 
18]. GDF15 is known to be involved in human embryo development and necessary for the maintenance of pregnancy [19]. GDF15 is also produced by adipocytes in response to oxidative stress [20], a well-known factor associated with preeclampsia.

In this study, we first determined the serum levels of GDF15 in the third trimester in women presenting with preeclampsia, and investigated whether the changes in GDF15 depend on preeclampsia subtype (early-onset vs late-onset). We then examined whether serum GDF15 is altered at 11-13 weeks of gestation in women who are destined to develop preeclampsia later in pregnancy. 


\section{Materials and Methods}

\section{Study population}

Maternal blood samples from preeclamptic women $(n=34)$ at diagnosis in the third trimester and direct gestation-age-matched normotensive pregnant women $(n=66)$ were collected by venipuncture into plain vaccutainer tubes before any clinical treatment. Preeclampsia samples were grouped into subtypes of early-onset ( $<34$ weeks, $n=16$; direct gestation-age-matched control $n=20$ ) and late-onset (>34 weeks, $n=18$; direct gestation-age-matched control $n=46$ ), and their clinical characteristics are summarized in Table 1. These blood samples were collected at the Department of Obstetrics, Wuxi Maternity and Children's Hospital, Nanjing Medical University, China; the collection was from February 2010 to January 2012. In addition, a few sera were separately collected in the same facilitate in 2014 from healthy pregnant women at gestation weeks of 11-13 (n=9), 23-27 $(n=9)$ and 33-40 (n=11); these served as representative normal pregnant sera of different trimesters to specifically analyse the GDF15 levels across gestation.

The blood was allowed to clot and centrifuged at $1500 \mathrm{~g}$, the serum was aspirated and stored in aliquots at $-80{ }^{\circ} \mathrm{C}$ until use. The study was approved by the Ethics Committee of Wuxi Maternity and Children's Hospital, Nanjing Medical University, China. All samples were obtained with written informed consent.

In addition, in order to investigate whether serum GDF15 levels were altered in the first trimester in women destined to develop preeclampsia, serum samples were also prospectively collected at 11-13 weeks of gestation from women who then proceeded with uncomplicated pregnancies or developed late-onset preeclampsia in the third trimester. These were collected at Royal Prince Alfred Hospital, Sydney, Australia, and participants were originally recruited to a study evaluating an algorithm for predicting the risk of pre-eclampsia in the first trimester $[21,22]$. For the current study, singleton pregnant sera representing normal 
pregnancy $(n=33)$ and pregnancies destined to develop late-onset preeclampsia $(n=36)$ were randomly selected from the above collection. The study was conducted in accordance with ethics approval from Royal Prince Alfred Hospital, Sydney, Australia (Study No. X11-0305 \& HREC/11/RPAH/472).

A number of studies suggested that the Preeclampsia was defined as maternal systolic blood pressure $\geq 140 \mathrm{mmHg}$ and/or diastolic blood pressure $\geq 90 \mathrm{mmHg}$ on two occasions separated by 6 hours, plus proteinuria $>300 \mathrm{mg}$ in a 24 hour period after 20 weeks of gestation; this was in accordance with the guidelines of the American College of Obstetricians and Gynaecologists [23]. Preeclampsia occurring earlier than 34 weeks of gestation was defined as early-onset, and those presenting after 34 weeks of gestation was defined as late-onset.

\section{Analysis of serum GDF15}

Serum levels of GFD15 were measured using a GDF15 ELISA kit (R\&D Systems, Minneapolis, MN, USA) following the manufacturer's instructions.

\section{Statistical analysis}

Due to differences in sample collection timing (more than 2 years apart), GDF15 levels were analyzed separately for different cohorts. Clinical data were presented as mean and standard deviation (SD). The GDF15 levels were also presented as mean \pm SD. The differences in GFD15 levels among the $1^{\text {st }}, 2^{\text {nd }}$ and $3^{\text {rd }}$ trimesters were analysed by ANOVA test (KruskalWallis test, because the data were not normally distributed). The differences in GFD15 between preeclampsia (either early-onset or late-onset subtypes) and gestation-age-matched normotensive controls were analysed by Mann-Whitney test as the data were not normally distributed either. All the statistical analyses were performed using the Prism software package (GraphPad Software, Inc, San Diego, CA, USA) and $\mathrm{P}<0.05$ was considered as 
statistically significant. 


\section{Results}

\section{Demographic information of the study population}

The clinical characteristics of the 34 sera that were collected at the time of preeclampsia presentation and the gestation-age-matched 66 control samples are summarised in Table 1. Among the 34 preeclampsia samples, 16 were from early-onset and 18 from late-onset subtypes.

The detailed characteristics of the 11-13 week sera that were collected long before the presentation of preeclampsia were previously published [21, 22]. This current study randomly selected 33 normal and 36 late-onset preeclampsia samples from nulliparous singleton pregnancies that had no history of chronic hypertension. The two groups (normal and lateonset preeclampsia) were not significantly different in maternal age, maternal body mass index, fetal birthweight or gestation at delivery.

\section{Serum levels of GDF15 increase with gestational age}

We first investigated whether the circulating levels of GDF15 are associated with gestational age in normotensive pregnant women. We determined GDF15 levels in sera collected in the first (11-13 weeks, $n=9)$, second (23-27 weeks, $n=9)$ and third (33-40 weeks, $n=11)$ trimesters of normal pregnancy. The circulating levels of GDF15 increased significantly as gestation advanced, reaching the highest level in $3^{\text {rd }}$ trimester (Figure $1, p=0.0003$, ANOVA KruskalWallis test). Post-hoc test showed that GDF15 levels were significantly different between $1^{\text {st }}$ and $2^{\text {nd }}$ trimesters, and between $1^{\text {st }}$ and $3^{\text {rd }}$ trimesters (Figure 1 ). However, the $2^{\text {nd }}$ and $3^{\text {rd }}$ trimesters were not significantly different (Figure 1).

\section{Serum GDF15 is significantly reduced in preeclampsia at disease presentation}


We next compared serum levels of GDF15 in the third trimester from women who were presenting with preeclampsia and who were normotensive. The levels of GDF15 were significantly lower in women with preeclampsia $(20560 \pm 37362 \mathrm{pg} / \mathrm{ml}, \mathrm{n}=34)$ compared with their normotensive counterparts $(59776 \pm 33858 \mathrm{pg} / \mathrm{ml}, \mathrm{n}=66)(\mathrm{p}=0.001)$.

We further investigated whether serum GDF15 alterations were associated with the timing of preeclampsia onset. When the preeclampsia samples were separated into early-onset $(<34$ weeks, $\mathrm{n}=16$ ) and late-onset (>34 weeks, $\mathrm{n}=18$ ) subtypes, GDF15 levels were still significantly lower in preeclampsia compared to their respective normotensive controls $(<34$ weeks, $n=20 ;>34$ weeks, n=46, Figure 2). However, it's notable that the reduction was more drastic and consistent for the late-onset than the early-onset subtype compared to their respective controls (Figure 2, early-onset, $\mathrm{p}=0.001$; late-onset, $\mathrm{p}=0.0001$ ).

As the difference in serum GDF15 was more pronounced for late-onset preeclampsia, we performed a receiver operating characteristic (ROC) analysis (Figure 3) for this sub-type $(n=18)$ and their respective control samples $(n=46)$ presented in Figure 2 The area under the ROC curve was $0.91(\mathrm{p}<0.0001)$; at a cutoff of $<37567 \mathrm{pg} / \mathrm{ml}$, the specificity was $96 \%$ and sensitivity was $89 \%$.

\section{First trimester serum GDF15 is not altered in women destined to develop late-onset preeclampsia}

We further investigated whether serum GDF15 is altered in the first trimester in women who are destined to develop late-onset preeclampsia in the third trimester. We screened for GDF15 in a total of 69 maternal sera collected at 11-13 weeks of gestation from women who later developed late-onset preeclampsia $(n=36)$ or proceeded with normal pregnancies $(n=33)$.

The two groups showed very similar levels of serum GDF15 (Figure 4, p=0.79), indicating 
that first trimester serum GDF15 has no predictive value for later development of preeclampsia. 


\section{Discussion}

GDF15 was initially isolated from activated human macrophages in 1997 [24] and subsequently shown to be highly expressed in the placenta [25, 26]. GDF15 is expressed in trophoblast cell columns that will differentiate into invasive extravillous trophoblast (EVT) [19], it is also expressed in the syncytiotrophoblast throughout pregnancy [19]. The circulating levels of GDF15 increase dramatically during early pregnancy, and the levels at 10-14 weeks of gestation are 15-fold higher in comparison to the non-pregnant state [27]. Lower levels of GDF15 in the first trimester are associated with miscarriage [28]. These data suggest that GDF15 may play important physiological roles in placental development and function. However, it remains unclear whether the circulating levels of GDF15 are altered in preeclampsia at the time of or prior to disease presentation.

In this study, we showed that the serum levels of GDF15 increased significantly with increasing gestation, reaching the highest level in the $3^{\text {rd }}$ trimester (33-40 weeks of gestation). We also found that the serum levels of GDF15 in the third trimester were significantly lower in women presenting with preeclampsia. A significant reduction in serum GDF15 was seen in late-onset as well as early-onset preeclampsia subtypes, but the reduction was more profound in late-onset cases. We thus further investigated whether serum GDF15 in the first trimester could predict later development of late-onset preeclampsia, however, no difference was found in serum GDF15 at 11-13 weeks of gestation between women who later developed late-onset preeclampsia and who had uncomplicated pregnancies.

The increase in circulating levels of GDF15 during pregnancy is attributed to placental expression [29]. In addition, because iron regulates the secretion of GDF15 [30] and iron absorption is increased during pregnancy [31], iron regulation may also contribute to the observed GDF15 serum changes in pregnancy. A previous study reported that the circulating levels of GDF15 peaked at 33-35 weeks of gestation [19], this is consistent with our 
observation that serum GDF15 increased with gestation and that the level reached the highest in the third trimester.

In this study, we found that the serum levels of GDF15 were significantly decreased in the third trimester in women presenting with preeclampsia compared to normotensive, and that this GDF15 reduction was not associated with the timing of preeclampsia onset. These results are different from two previous reports [19, 32]. In the study of Marjono et al [19], no difference in serum levels of GDF15 was found between preeclamptic and control groups in the third trimester. On the other hand, the study of Sugulle et al reported a significant increase in circulating levels of GDF15 in preeclamptic women at term (gestational week $\geq 37$ ) compared to gestation age-matched normotensive controls [32]. The exact reasons for these differences are unknown; however, differences in sample size, maternal age and ethnicities may be contributing factors. The sample size of preeclampsia in Marjono's and Sugulle' study was 23 and 21 respectively, while our current study had a total of 34 women with preeclampsia. In addition, circulating levels of GDF15 are reported to positively associate with age over 45 years $[33,34]$. The median maternal age of our study was 26 (control) and 27 (preeclampsia) respectively, whereas the median maternal age studied by Sugulel et al was 33 (control) and 31 (preeclampsia) years respectively. But the maternal age for the Marjono's subjects was not reported [19]. Another reason could be that we and Marjono et al [19] analyzed serum, whereas plasma was assayed by Sugulle et al [32]. Using plasma vs serum may give different results, as a number of studies indicate that serum may differ from plasma in the detection of preeclampsia [35]. Ethnicity has been suggested to be associated with the pathogenesis of preeclampsia [36, 37], therefore the ethnicity difference may be a contributing factor to the result discrepancies between our study and that of Sugulle et al [32]. However, these mentioned differences do not fully explain the observed differences in GDF15 levels, and future studies are needed to address and clarify these. 
Furthermore, our current study further analyzed preeclampsia into early-onset and late-onset subtypes, which was not considered by the previous two studies $[19,32]$. Our data shows that the reduction in serum GDF15 was more severe in late-onset than early-onset cases, highlighting that the two preeclampsia subtypes are distinct.

Future studies need to investigate whether a reduction in serum GDF15 contributes to the development of preeclampsia, or it is a maternal response to preeclampsia. However, we found no difference in serum GDF15 levels at 11-13 weeks of gestation between women who later developed late-onset preeclampsia and who did not. This data suggests that serum GDF15 may not influence the causes of preeclampsia development. It's likely that the reduction in serum GDF15 seen at the time of preeclampsia presentation is due to maternal response to the disease. Although we were not able to assess whether serum levels of GDF15 in first trimester are altered in women who later develop early-onset preeclampsia, our study does not suggest that the first trimester serum GDF15 will be useful to predict future development of late-onset preeclampsia. However, it will be interesting to determine whether GDF15 levels in second trimester would be useful to predict future development of preeclampsia.

In conclusion, we demonstrated that in normal pregnancy the circulating levels of GDF15 increased with gestational age and that the GDF15 level was highest in $3^{\text {rd }}$ trimester. We also found that serum levels of GDF15 were significantly reduced in women presenting with preeclampsia in the third trimester, and that this reduction was more profound in late-onset than early-onset cases. However, serum GDF15 was not altered at 11-13 weeks of gestation in women who later developed late-onset preeclampsia. 


\section{Acknowledgments}

This study was supported by the Chinese National Nature Sciences Foundation (Grant No. 81100437 to M Zhao), the National Health and Medical Research Council of Australia (Fellowship \#1041835 and project grant \#1108365 to GN), the Bill and Melinda Gates Foundation, and the Victorian Government Operational Infrastructure Support Program in Australia. Authors would also like to thank all the patients who donated their blood samples for this study. 


\section{References}

1. Sibai B, Dekker G, Kupferminc M. Pre-eclampsia. The Lancet. 2005;365(9461):78599.

2. Roberts J, Taylor R, Musci T, Rodgers G, Hubel C, McLaughlin M. Preeclampsia: an endothelial cell disorder. American journal of obstetrics and gynecology. 1989;161(5):1200.

3. Redman CW, Sargent IL. Latest advances in understanding preeclampsia. Science. 2005;308(5728):1592-4.

4. von Dadelszen P, Magee LA, Roberts JM. Subclassification of preeclampsia. Hypertens Pregnancy. 2003;22(2):143-8.

5. Bulletins_Obstetrics ACoP. Diagnosis and Managment of Preeclampsia amd Eclampsia. Obstet Gynecol. 2001(99):159.

6. Teoh SS, Zhao M, Wang Y, Chen Q, Nie G. Serum HtrA1 is differentially regulated between early-onset and late-onset preeclampsia. Placenta. 2015;36(9):990-5.

7. Singh H, Zhao M, Chen Q, Wang Y, Li Y, Kaitu'u-Lino TJ, et al. Human HtrA4 Expression Is Restricted to the Placenta, Is Significantly Up-Regulated in Early-Onset Preeclampsia, and High Levels of HtrA4 Cause Endothelial Dysfunction. J Clin Endocrinol Metab. 2015;100(7):E936-45.

8. Burton GJ, Hempstock J, Jauniaux E. Oxygen, early embryonic metabolism and free radical-mediated embryopathies. Reprod Biomed Online. 2003;6(1):84-96.

9. Bellamy L, Casas JP, Hingorani AD, Williams DJ. Pre-eclampsia and risk of cardiovascular disease and cancer in later life: systematic review and meta-analysis. BMJ. 2007;335(7627):974.

10. Harskamp RE, Zeeman GG. Preeclampsia: at risk for remote cardiovascular disease. The American journal of the medical sciences. 2007;334(4):291-5. 
11. Barden A. Pre-eclampsia: contribution of maternal constitutional factors and the consequences for cardiovascular health. Clin Exp Pharmacol Physiol. 2006;33(9):826-30. 12. Zimmers TA, Jin X, Hsiao EC, McGrath SA, Esquela AF, Koniaris LG. Growth differentiation factor-15/macrophage inhibitory cytokine-1 induction after kidney and lung injury. Shock. 2005;23(6):543-8.

13. Adela R, Banerjee SK. GDF-15 as a Target and Biomarker for Diabetes and Cardiovascular Diseases: A Translational Prospective. Journal of diabetes research. 2015;2015:490842.

14. Ferrari N, Pfeffer U, Dell'Eva R, Ambrosini C, Noonan DM, Albini A. The transforming growth factor-beta family members bone morphogenetic protein- 2 and macrophage inhibitory cytokine- 1 as mediators of the antiangiogenic activity of $\mathrm{N}$-(4hydroxyphenyl)retinamide. Clin Cancer Res. 2005;11(12):4610-9.

15. Xu J, Kimball TR, Lorenz JN, Brown DA, Bauskin AR, Klevitsky R, et al. GDF15/MIC-1 functions as a protective and antihypertrophic factor released from the myocardium in association with SMAD protein activation. Circ Res. 2006;98(3):342-50. 16. Yokoyama-Kobayashi M, Saeki M, Sekine S, Kato S. Human cDNA encoding a novel TGF-beta superfamily protein highly expressed in placenta. J Biochem. 1997;122(3):622-6.

17. Tan M, Wang Y, Guan K, Sun Y. PTGF-beta, a type beta transforming growth factor (TGF-beta) superfamily member, is a p53 target gene that inhibits tumor cell growth via TGF-beta signaling pathway. Proc Natl Acad Sci U S A. 2000;97(1):109-14.

18. Koopmann J, Buckhaults P, Brown DA, Zahurak ML, Sato N, Fukushima N, et al. Serum macrophage inhibitory cytokine 1 as a marker of pancreatic and other periampullary cancers. Clin Cancer Res. 2004;10(7):2386-92. 
19. Marjono AB, Brown DA, Horton KE, Wallace EM, Breit SN, Manuelpillai U. Macrophage inhibitory cytokine-1 in gestational tissues and maternal serum in normal and pre-eclamptic pregnancy. Placenta. 2003;24(1):100-6.

20. Ding Q, Mracek T, Gonzalez-Muniesa P, Kos K, Wilding J, Trayhurn P, et al. Identification of macrophage inhibitory cytokine- 1 in adipose tissue and its secretion as an adipokine by human adipocytes. Endocrinology. 2009;150(4):1688-96.

21. Park FJ, Leung CH, Poon LC, Williams PF, Rothwell SJ, Hyett JA. Clinical evaluation of a first trimester algorithm predicting the risk of hypertensive disease of pregnancy. Aust N Z J Obstet Gynaecol. 2013;53(6):532-9.

22. Cnossen JS, Vollebregt KC, de Vrieze N, ter Riet G, Mol BW, Franx A, et al. Accuracy of mean arterial pressure and blood pressure measurements in predicting preeclampsia: systematic review and meta-analysis. BMJ. 2008;336(7653):1117-20.

23. Hypertension in Pregnancy. American College of Obstetricians and Gynecologists Women's Health Care Physician. 2013;November.

24. Bootcov MR, Bauskin AR, Valenzuela SM, Moore AG, Bansal M, He XY, et al. MIC-1, a novel macrophage inhibitory cytokine, is a divergent member of the TGF-beta superfamily. Proc Natl Acad Sci U S A. 1997;94(21):11514-9.

25. Lawton LN, Bonaldo MF, Jelenc PC, Qiu L, Baumes SA, Marcelino RA, et al. Identification of a novel member of the TGF-beta superfamily highly expressed in human placenta. Gene. 1997;203(1):17-26.

26. Fairlie WD, Moore AG, Bauskin AR, Russell PK, Zhang HP, Breit SN. MIC-1 is a novel TGF-beta superfamily cytokine associated with macrophage activation. J Leukoc Biol. 1999;65(1):2-5.

27. Moore AG, Brown DA, Fairlie WD, Bauskin AR, Brown PK, Munier ML, et al. The transforming growth factor-ss superfamily cytokine macrophage inhibitory cytokine-1 is 
present in high concentrations in the serum of pregnant women. J Clin Endocrinol Metab. $2000 ; 85(12): 4781-8$

28. Tong S, Marjono B, Brown DA, Mulvey S, Breit SN, Manuelpillai U, et al. Serum concentrations of macrophage inhibitory cytokine 1 (MIC 1) as a predictor of miscarriage. Lancet. 2004;363(9403):129-30.

29. Hromas R, Hufford M, Sutton J, Xu D, Li Y, Lu L. PLAB, a novel placental bone morphogenetic protein. Biochim Biophys Acta. 1997;1354(1):40-4.

30. Tanno T, Bhanu NV, Oneal PA, Goh SH, Staker P, Lee YT, et al. High levels of GDF15 in thalassemia suppress expression of the iron regulatory protein hepcidin. Nat Med. 2007;13(9):1096-101.

31. Millard KN, Frazer DM, Wilkins SJ, Anderson GJ. Changes in the expression of intestinal iron transport and hepatic regulatory molecules explain the enhanced iron absorption associated with pregnancy in the rat. Gut. 2004;53(5):655-60.

32. Sugulle M, Dechend R, Herse F, Weedon-Fekjaer MS, Johnsen GM, Brosnihan KB, et al. Circulating and placental growth-differentiation factor 15 in preeclampsia and in pregnancy complicated by diabetes mellitus. Hypertension. 2009;54(1):106-12.

33. Wiklund FE, Bennet AM, Magnusson PK, Eriksson UK, Lindmark F, Wu L, et al. Macrophage inhibitory cytokine-1 (MIC-1/GDF15): a new marker of all-cause mortality. Aging cell. 2010;9(6):1057-64.

34. Corre J, Hebraud B, Bourin P. Concise review: growth differentiation factor 15 in pathology: a clinical role? Stem cells translational medicine. 2013;2(12):946-52.

35. Smarason AK, Sargent IL, Redman CW. Endothelial cell proliferation is suppressed by plasma but not serum from women with preeclampsia. Am J Obstet Gynecol. 1996;174(2):787-93. 
36. Anderson NH, McCowan LM, Fyfe EM, Chan EH, Taylor RS, Stewart AW, et al. The impact of maternal body mass index on the phenotype of pre-eclampsia: a prospective cohort study. BJOG. 2012;119(5):589-95.

37. Xiao J, Shen F, Xue Q, Chen G, Zeng K, Stone P, et al. Is ethnicity a risk factor for developing preeclampsia? An analysis of the prevalence of preeclampsia in China. J Hum Hypertens. 2014;28(11):694-8. 
Table 1. Clinical characteristics of the cohort used for analyzing serum GDF15 at preeclampsia presentation

\begin{tabular}{|l|l|l|l|}
\hline & Preeclampsia $(\mathrm{n}=34)$ & Normotensive $(\mathrm{n}=66)$ & $\mathrm{P}$-value \\
\hline Maternal age (years, mean/SD) & $28.2 \pm 5.3$ & $26.1 \pm 3.7$ & $\mathrm{P}=0.116$ \\
\hline Gestational age at diagnosis & $32^{+2} \pm 5$ & NA & $\mathrm{NA}$ \\
\hline Systolic $(\mathrm{mmHg}$, mean/SD) & $157 \pm 13$ & $122 \pm 8.8$ & $\mathrm{p}<0.0001$ \\
\hline Diastolic $(\mathrm{mmHg}, \mathrm{mean} / \mathrm{SD})$ & $106 \pm 14$ & $78 \pm 6.5$ & $\mathrm{p}<0.0001$ \\
\hline Proteinuria & $>1+$ & Negative & $\mathrm{NA}$ \\
\hline
\end{tabular}




\section{Figure legends}

Figure 1: The serum levels of GDF15 increase with gestational age in normal pregnancy. Serum GDF15 was determined by ELISA in maternal sera collected in the $1^{\text {st }}(n=9), 2^{\text {nd }}(n=9)$ and $3^{\text {rd }}(\mathrm{n}=11)$ trimesters. Data are expressed as mean \pm SD. ANOVA (Kruskal-Wallis test) and Post-hoc test were performed.

Figure 2: Serum GDF15 is significantly decreased in both early-onset and late-onset preeclampsia at disease presentation. Preeclampsia was divided into early-onset $(<34$ weeks, $n=16$ ) and late-onset (>34 weeks, $n=18$ ) subtypes, and serum GDF15 levels were compared to their respective gestation-age-matched normotensive controls $(<34$ weeks, $n=20 ;>34$ weeks, $n=46$ ). Data are expressed as mean $\pm S D$, Mann-Whitney test was performed.

Figure 3: Receiver-operating characteristic (ROC) curve of serum GDF15 for late-onset preeclampsia at disease presentation. The data of late-onset ( $>34$ weeks, $n=18$ ) preeclampsia and the gestation-age-matched normotensive controls $(n=46)$ presented in Figure 2 were analysed. The area under the curve was 0.91 with $95 \%$ confidence interval of $0.81-1.02, \mathrm{p}<0.0001$.

Figure 4: The serum levels of GDF15 at 11-13 weeks of gestation are not altered in women who are destined to develop late-onset preeclampsia. Serum GDF15 levels at 1113 weeks of gestation were compared between women who later developed late-onset preeclampsia $(n=33)$ and who proceeded with uncomplicated pregnancies $(n=36)$. Data are expressed as mean $\pm \mathrm{SD}$, Mann-Whitney test was performed. 
Figure 1

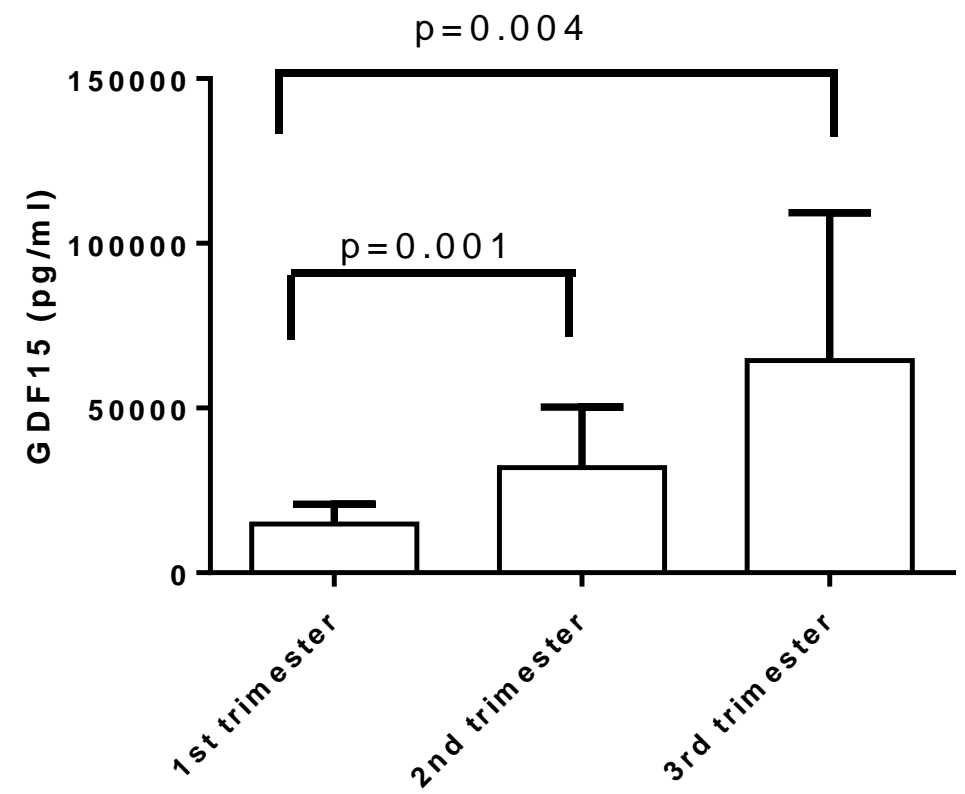

Figure 2

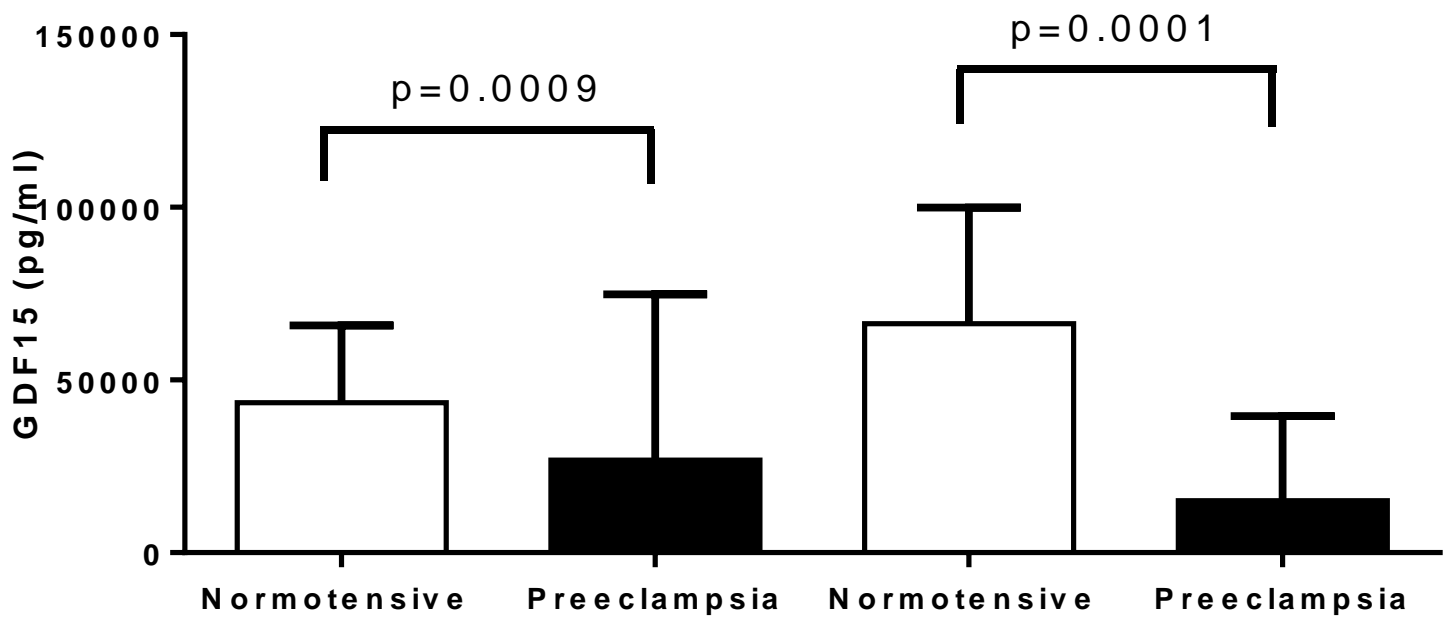

Early-onset ( $\leq 34$ weeks) Late-onset ( $>34$ weeks) 
Figure 3

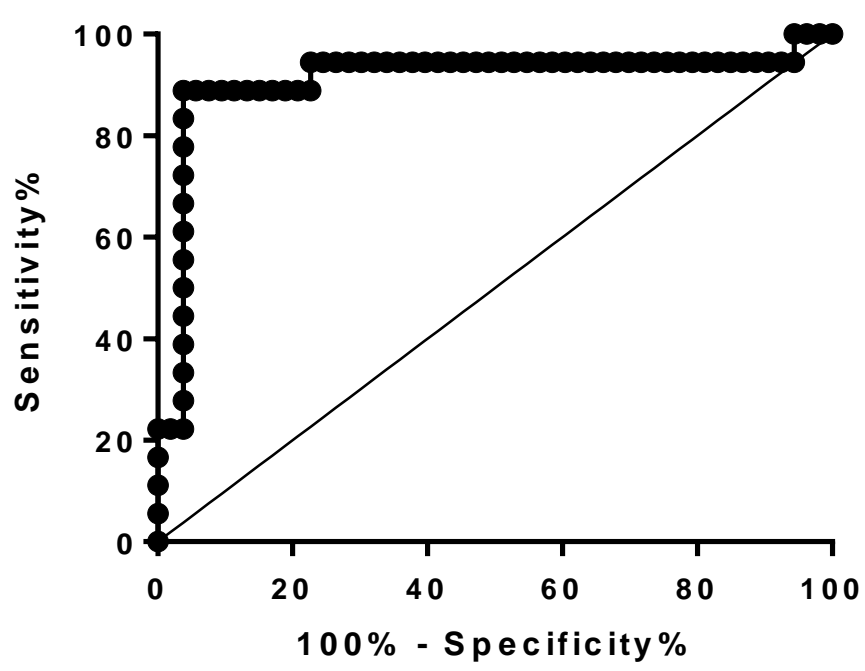

Figure 4

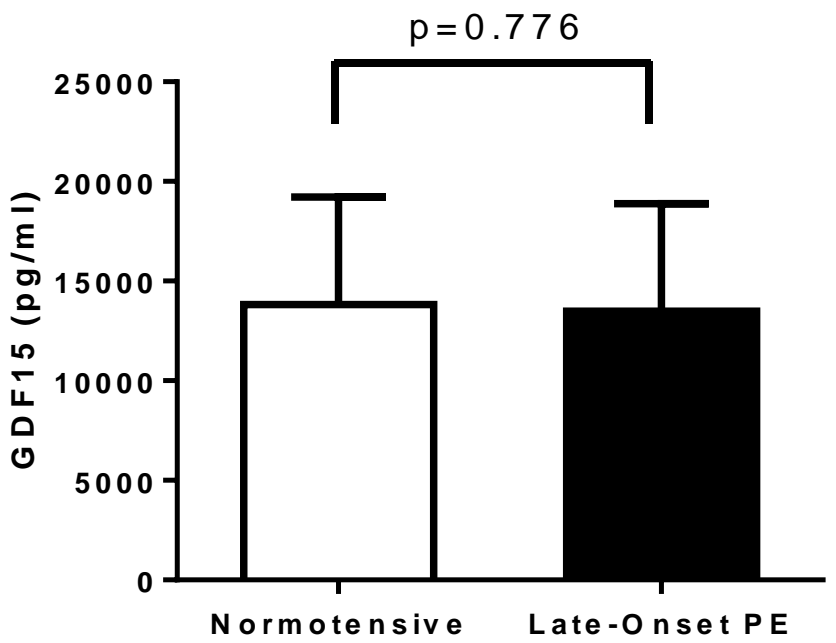

